\title{
Y-junction power splitter enhanced through subwavelength metamaterials
}

\author{
Raquel Fernández de Cabo \\ Instituto de Óptica \\ Consejo Superior de \\ Investigaciones Científicas \\ Madrid, Spain \\ r.fernandez@csic.es
}

\author{
David González-Andrade \\ Instituto de Óptica \\ Consejo Superior de \\ Investigaciones Cientificas \\ Madrid, Spain \\ david.gonzalez@csic.es
}

\author{
Pavel Cheben \\ Institute for Microstructural \\ Sciences \\ National Research Council \\ Canada \\ Ottawa, Canada \\ Pavel.Cheben@nrc-cnrc.gc.ca
}

\author{
Aitor V. Velalsco \\ Instituto de Óptica \\ Consejo Superior de \\ Investigaciones Científicas \\ Madrid, Spain \\ a.villafranca@csic.es
}

\begin{abstract}
We present a Y-junction beam splitter based on subwavelength metamaterials that circumvents losses associated to the fabrication limitations in the tip size of traditional Yjunctions. Simulations show excess losses under $0.5 \mathrm{~dB}$ for $\mathrm{TE}_{0}$ and $\mathrm{TE}_{1}$ in a $300 \mathrm{~nm}$ range $(1300-1600 \mathrm{~nm})$.
\end{abstract}

Keywords-Photonic integrated circuits, Silicon photonics, Power division, Y-junction, Subwavelength, Minimum feature size.

\section{INTRODUCTION}

Efficient power splitting is a fundamental need in siliconbased photonic integrated circuits (PICs) [1], with applications in mode-division multiplexing [2], optical phased arrays [3], or microspectrometers [4], to name a few. Y-junctions, consisting of a stem waveguide which branches into two diverging arms, are one of the most widespread beam splitters [5]. In symmetrical configurations, Y-junction theoretically work as a perfect power divider for both the fundamental mode and the first-order mode. However, the finite resolution of current micro fabrication methods results in a limited minimum feature size (MFS) of the junction tip between the splitter arms [5]. This deviation from the nominal design particularly penalizes fundamental mode losses, as their energy peak is located in the central region.

In order to reduce losses and improve bandwidth of PIC components, subwavelength gratings (SWG) were proposed [6]. SWG are segmented waveguides with a grating period $(\Lambda)$ significantly smaller than the wavelength $(\lambda)$ of propagating light. Under this condition, the medium behaves as a homogeneous metamaterial [7], whose optical properties (e.g. effective index, dispersion, anisotropy) can be tailored through geometric design. This approach has been successfully applied to mode-division multiplexing and microspectrometers [2, 4], among many others.

In this work, we propose the application of SWG technology to Y-junctions, effectively reducing mode confinement around the junction tip and hence circumventing minimum feature size penalization on the fundamental mode. Our device exhibits simulated excess losses (EL) as low as $0.5 \mathrm{~dB}$ for the fundamental transverse electric mode $\left(\mathrm{TE}_{0}\right)$ and the first-order transverse electric mode $\left(\mathrm{TE}_{1}\right)$ in a $300 \mathrm{~nm}$ bandwidth (1300 $1600 \mathrm{~nm})$.

\section{DEVICE OPERATION}

In order to compare our solution to a traditional approach, we consider as a reference a non-periodic Y-junction, whose scheme is shown in Fig. 1(a). This device comprises a multimode input stem of width $W_{0}$ and length $L_{s}$, and two monomode S-shaped output arms of width $W=W_{0} / 2$, length $L_{B}$. and final separation $H_{a}$. We consider a gap between the arms at the junction tip, of width $H_{\text {off }}$ which accounts for the MFS limitation in the fabrication process. A taper of length $L_{T}$ at the input and straight section of length $L o$ at the output are also included. Exciting the stem with $\mathrm{TE}_{1}$ mode results in almost loss-free power splitting as a consequence of the zero-power profile of this mode through the center of the stem, but losses are significantly increased for $\mathrm{TE}_{0}$ mode in the presence of the junction gap, further increasing the larger $H_{\text {off }}$ is.

Our device incorporates SWG metamaterials to both the arms and the stem, while preserving the same arm offset, and hence the same MFS (Fig. 1(b)). Arm widths and separation are also maintained. The device starts with a strip waveguide of length $L_{I}$ and width $W_{S}$ that evolves into a SWG waveguide of length $L_{C}$ through an adiabatic taper. This SWG region induces
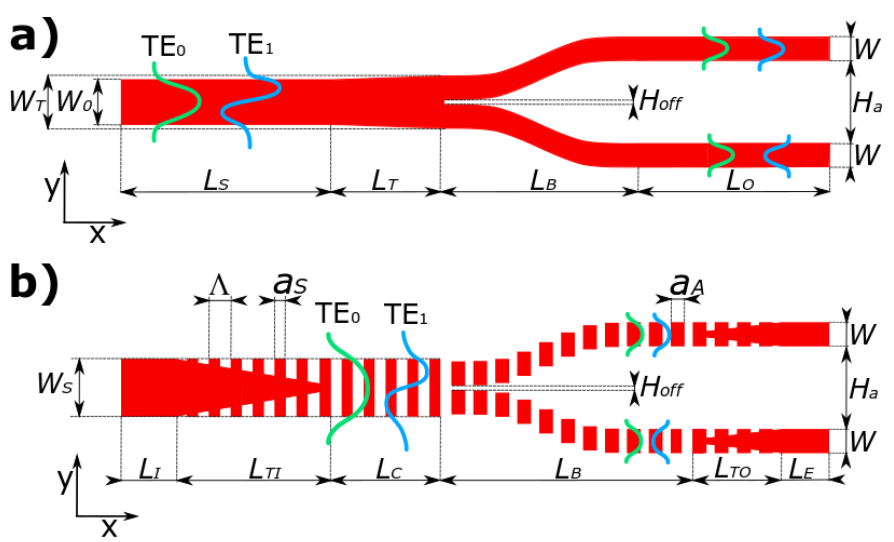

Fig. 1. Schematic of the state-of-the-art non-periodic (a) and the proposed SWG Y-junction (b). Propagation of fundamental and first-order modes along the device is also shown.

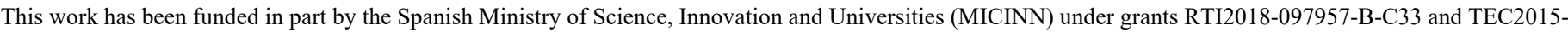

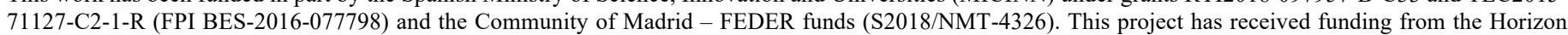
2020 research and innovation program under Marie Sklodowska-Curie grant No. 734331. 
a lesser mode confinement in order to reduce the radiation in the junction tip for $\mathrm{TE}_{0}$. Another advantage of $\mathrm{SWG}$ is the possibility to perform dispersion engineering through the modification of the pitch and the silicon segments length. Moreover, to optimize transmission in the interface between the stem and the arms, we define different duty cycles on both sides, $D C_{S}=a_{S} / \Lambda$ and $D C_{A}=a_{A} / \Lambda$, where $a_{S}$ is the silicon segment length in the stem and $a_{A}$ is the silicon segment length in the arms, respectively, considering a constant period.

\section{DEVICE DESIGN}

We optimized our device for a silicon-on-insulator platform with a waveguide core thickness of $220 \mathrm{~nm}$, with upper and bottom silicon dioxide cladding. The refractive index of each material is approximately $n_{\mathrm{Si}} \sim 3.48$ and $n_{\mathrm{SiO} 2} \sim 1.44$, for a wavelength of $\lambda \sim 1.55 \mu \mathrm{m}$. An arm width of $W=500 \mathrm{~nm}$ is selected in order to ensure the arms are monomode, while a stem width of $W_{s}=1200 \mathrm{~nm}$ provides an increased effective index of the stem that reduces interface reflections. An SWG pitch of $\Lambda=220 \mathrm{~nm}$ is set to avoid Bragg-reflection, while a $D C_{S}$ of $50 \%$ facilitates the fabrication process. We consider a $H_{\text {off }}$ of $100 \mathrm{~nm}$ for the worst-case scenario regarding manufacturing accuracy limitations, preserving this same limit as MFS for the whole design. A complete list of the others geometrical design parameters can be found in Table I.

TABLE I. PARAMETER VALUES

\begin{tabular}{|c|c|c|c|}
\hline Design & Parameter & Symbol & Value \\
\hline \multirow{3}{*}{$\begin{array}{lr}\text { SWG } & \\
\text { and } & \text { Non- } \\
\text { periodic } & \text { Y- } \\
\text { junction } & \end{array}$} & Arms width & $W$ & $0.5 \mu \mathrm{m}$ \\
\hline & Arms output separation & $H_{a}$ & $1.5 \mu \mathrm{m}$ \\
\hline & Arm length & $L_{B}$ & $9.8 \mu \mathrm{m}$ \\
\hline \multirow{4}{*}{$\begin{array}{l}\text { Non-periodic Y- } \\
\text { junction }\end{array}$} & Stem guide length & $L_{S}$ & $13 \mu \mathrm{m}$ \\
\hline & Taper length & $L_{T}$ & $4 \mu \mathrm{m}$ \\
\hline & Output-section length & $L_{O}$ & $9 \mu \mathrm{m}$ \\
\hline & Taper final width & $W_{T}$ & $1.1 \mu \mathrm{m}$ \\
\hline \multirow{6}{*}{$\begin{array}{l}\text { SWG } \\
\text { Y-junction }\end{array}$} & Input strip width & $W_{S}$ & $1.2 \mu \mathrm{m}$ \\
\hline & Input strip length & $L_{I}$ & $3 \mu \mathrm{m}$ \\
\hline & Input SWG taper & $L_{T I}$ & $10 \mu \mathrm{m}$ \\
\hline & Output SWG taper & $L_{T O}$ & $6 \mu \mathrm{m}$ \\
\hline & Central SWG section & $L_{C}$ & $13 \mu \mathrm{m}$ \\
\hline & Output strip length & $L_{E}$ & $3 \mu \mathrm{m}$ \\
\hline
\end{tabular}

\section{SimULATION RESUlTS}

The device performance was simulated through 3D-FDTD simulation. In order to optimize excess losses (EL), i.e. the relation between the optical power at the output arms in relation to the power injected in the input stem, we swept $D C_{A}$ while holding $D C_{S}$ constant. We found minimum EL for $\mathrm{TE}_{1}$ at $D C_{A}$ $=55 \%$, while $\mathrm{EL}$ for $\mathrm{TE}_{0}$ presents a flatter response against $D C_{A}$ starting around the same value. Note that this DC value imposes a separation between SWG segments in the arms of $100 \mathrm{~nm}$, thus respecting MFS.

Excess loss obtained for the SWG device and for the reference non-periodic counterpart are shown in Fig. 2. Our

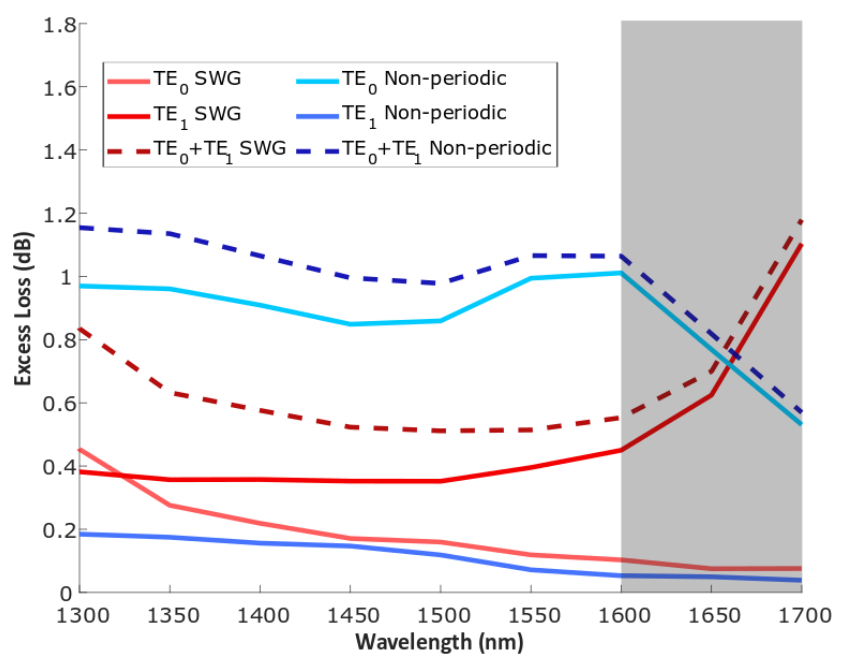

Fig. 2. Excess loss comparision between SWG and non-periodic Yjunction for $\mathrm{TE}_{0}$ and $\mathrm{TE}_{1}$ modes.

design exhibits a significant reduction in $\mathrm{TE}_{0}$ excess losses at the nominal wavelength of $1550 \mathrm{~nm}$ (from $0.99 \mathrm{~dB}$ down to 0.12 $\mathrm{dB}$ ). Despite a slight increase of $\mathrm{TE}_{1}$ excess losses (from 0.07 $\mathrm{dB}$ up to $0.40 \mathrm{~dB}$ ), the sum of both EL values is significantly smaller in the SWG device, which also provides a more even performance for both modes. This behavior is preserved in a very broad bandwidth, as simulations show EL under $0.5 \mathrm{~dB}$ for both $\mathrm{TE}_{0}$ and $\mathrm{TE}_{1}$ in a $300 \mathrm{~nm}$ bandwidth $(1300-1600 \mathrm{~nm})$.

\section{CONCLUSIONS}

We have proposed a high-performance power splitter based on a Y-junction enhanced through subwavelength metamaterials, which reduces fundamental mode losses caused by fabrication limitations at the junction tip. Simulations show insertion losses as low as $0.5 \mathrm{~dB}$ for $\mathrm{TE}_{0}$ and $\mathrm{TE}_{1}$ in a $300 \mathrm{~nm}$ bandwidth $(1300-1600 \mathrm{~nm})$. We believe that the proposed optical beam splitter will find numerous applications in diverse photonic integrated circuits, and particularly, in mode-division multiplexing applications.

\section{REFERENCES}

[1] L. Chrostowski, et al., Silicon photonics design: from devices to systems, Cambridge University Press, 2015.

[2] D. González-Andrade, et al., "Ultra-Broadband Mode Converter and Multiplexer Based on Sub-Wavelength Structures," IEEE Photon. J., vol. 10, p. 2201010, 2018.

[3] J. K. Doylend, et al, "Two-dimensional free-space beam steering with an optical phased array on silicon-on-insulator, "Opt. Express, vol. 19, pp. 21595-21604, 2011.

[4] A. Herrero-Bermello, et al., "On-chip Fourier-transform spectrometers and machine learning: a new route to smart photonic sensors," Opt. Lett., vol. 44, pp. 5840-5843, 2019.

[5] Y. Zhang, et al., "A compact and low loss Y-junction for submicron silicon waveguide," Opt. Express, vol. 21, pp. 1310-131, 2013.

[6] P. Cheben, et al., "Subwavelength integrated photonics," Nature, vol. 560, pp. 565-572, 2018.

[7] P. Lalanne, et al., "High-order effective-medium theory of subwavelength gratings in classical mounting: application to volume holograms," J. Opt. Soc. Am. A., vol. 15, pp.1843-1851, 1998. 